\title{
Glutathione Peroxidase-Activatable Two-Photon Ratiometric Fluorescent Probe for Redox Mechanism Research in Aging and Mercury Exposure Mice Models
}

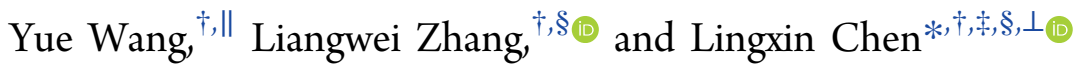 \\ ${ }^{\dagger}$ CAS Key Laboratory of Coastal Environmental Processes and Ecological Remediation, Research Center for Coastal Environmental \\ Engineering and Technology, Yantai Institute of Coastal Zone Research, Chinese Academy of Sciences, Yantai 264003, China \\ ${ }^{\ddagger}$ School of Pharmacy, Binzhou Medical University, Yantai 264003, China \\ ${ }^{\S}$ Center for Ocean Mega-Science, Chinese Academy of Sciences, Qingdao 266071, China \\ "University of Chinese Academy of Sciences, Beijing 100049, China \\ ${ }^{\perp}$ Department of Chemistry and Chemical Engineering, Qufu Normal University, Qufu 273165, China
}

\section{Supporting Information}

\begin{abstract}
Solid evidence confirms that glutathione peroxidase (GPx) is a kind of vital protease in the first-line antioxidant defense system and participates in regulation of redox homeostasis as well as the pentose phosphate pathway. However, the current methods cannot achieve real-time and in situ visualization studies of GPx. In addition, GPx is highly reactive and susceptible to external interference, and there is rare research for exploring the roles of GPx under environmental factor exposure. Herein, we report a novel two-photon ratiometric fluorescent probe (TP-SS) for GPx detection for

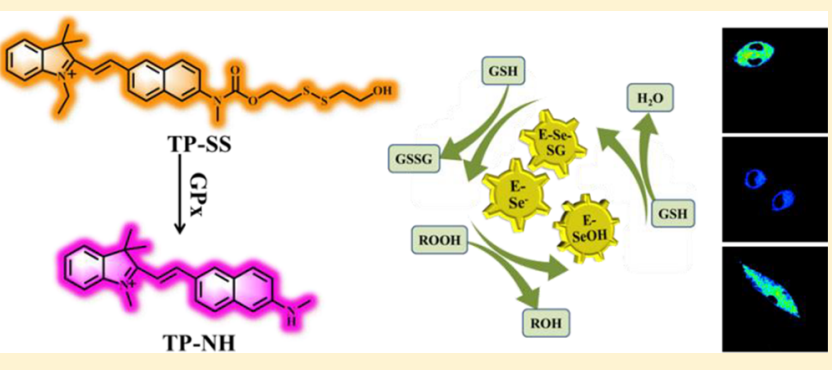
the first time. Using TP-SS, we explore the reversible catalytic cycle and the antioxidant mechanisms of GPx/GSH redox pool in aging and mercury exposure models. We detect the concentration fluctuation of GPx in aging and mercury exposure mice models. Also, we perform GPx detection in deep brain tissue and the imaging depth up to $100 \mu \mathrm{m}$. We believe that the novel two-photon ratiometric fluorescent probe TP-SS can facilitate the development of GPx-targeting tools and offer great advances in exploring the physiological/pathological functions of GPx.
\end{abstract}

$\mathrm{T}$ he glutathione peroxidase/glutathione (GPx/GSH) redox pool consists of glutathione peroxidases (GPx), glutathione (GSH), and a series of cofactors. ${ }^{1,2}$ As a cental member, $\mathrm{GPx}$ is a kind of selenocysteine ( $\mathrm{Sec}$ )-containing protein which plays significant roles ranging from regulation of redox homeostasis and the pentose phosphate pathway to vascular tone. $^{3-5}$ Selenoproteins often incorporate into proteins as the active sites in vivo, for example, the active site of GPx is Sec. ${ }^{1-3}$ The GPx/GSH redox pool may be the first line volunteer in the cellular defense system against oxidative stress which considered being Sec previously. ${ }^{6}$ Excessive reactive oxygen species (ROS) will cause an imbalance in the redox system. In the GPx/GSH redox pool, GPx can reduce ROS by means of a bisubstrate ping-pong-type enzymatic mechanism for ROS detoxification. ${ }^{2}$ Lacking of selenium will cause an imbalance in the redox system and hinder biosynthesis of physiologically relevant biomacromolecules, which will further cause several diseases, for example neurodegenerative diseases, cardiovascular diseases, and cancers. Therefore, it is essential to maintain the balance of GPx/GSH redox pool, which influences the homeostasis in organism milieu. ${ }^{7}$ Despite GPx playing vital roles in vivo especially in the antioxidant defense systems, there is no available tool for visually tracking GPx up to now. Great efforts on this aspect should be devoted to developing reliable, rapid, and visual tools for GPx detection.

Research shows that oxidative stress during the process of aging becames more sever. ${ }^{8}$ The free radicals theory in aging suggests that aging and some related diseases are the results of oxidative stress. ${ }^{9}$ Thus, the GPx/GSH redox pool, an important antioxidant system, plays vital roles in preventing diseases and reducing in the speed of aging. ${ }^{8}$ Exploring the cyclic mechanism of $\mathrm{GPx} / \mathrm{GSH}$ redox pool can provide important insights into the biological basis of increased oxidative stress in aging humans and into potential targets for clinical intervention. ${ }^{10}$ In addition, organisms are undergoing severe ordeals with the ecological environment deteriorates. Heavy metal ions, especially the mercury ion $\left(\mathrm{Hg}^{2+}\right)$, have destructive effects on organisms. $\mathrm{Hg}^{2+}$ can easily move into organisms through biomembranes and cause the organism injury by combining to proteins with physiological functions as well as further cause the outbreak of ROS. ${ }^{11}$

Received: September 25, 2019

Accepted: December 20, 2019

Published: December 20, 2019 
Studies show that $\mathrm{Hg}^{2+}$ has a strong affinity to selenoprotein such as Sec. ${ }^{11}$ Thus, $\mathrm{Hg}^{2+}$ may destroy the activity of proteases especially GPx. ${ }^{12}$ Exploring the interactions between GPx/ GSH redox pool and $\mathrm{Hg}^{2+}$ are much more meaningful for elucidating the physiopathological mechanisms associated with $\mathrm{GPx} / \mathrm{GSH}$ redox pool and mercury poisoning.

Traditional methods for GPx detection are based on gel electrophoresis protocols, matrix-assisted laser desorption ionization/electrospray ionization mass spectrometry, and Western blot. ${ }^{13-15}$ However, the samples pretreatment needs to destroy tissues or cells, and the manipulation is very complicated during GPx detection. More importantly, all of these methods cannot achieve real-time and in situ detection. To the best of our knowledge, there are no convenient methods for GPx visualization detection in real-time and in situ, which limits the studies of the key oxidoreductase. Fluorescence imaging technology which owns rapid response, highly selectivity, less invasiveness, and high spatial and temporal resolution has been widely used for detection of various active species in vivo. ${ }^{11}$ Furthermore, two-photon functional fluorescent probes that absorb two near-infrared (NIR) photons as the excitation source have many advantages than one-photon fluorescent probes, for example prolonged observation time, the capability of imaging in deep tissues, and higher resolution. ${ }^{16}$ Thus, a highly selective two-photon fluorescent probe is needed urgently for GPx detection.

Herein, we designed and synthesized a two-photon functional fluorescent probe, TP-SS, for GPx detection. Using TPSS, we investigated the concentration fluctuations of GPx and the reversible catalytic mechanism of GPx/GSH redox pool in aging and mercury poisoning cell models. The cell damages caused by oxidative stress and $\mathrm{Hg}^{2+}$ were evaluated by Annexin V/7-AAD Apoptosis Detection Kit, JC-1, and transmission electron microscope (TEM). We also performed GPx detection in aging and mercury poisoning mice models. The tissue damages were assess by H\&E and TUNEL staining. We further performed GPx detection in deep brain tissue, and the imaging depth up to $100 \mu \mathrm{m}$. To the best of our knowledge, TP-SS is the first fluorescent visualization tool for imaging and quantifying the protective effects of GPx in cells and in vivo during aging and mercury poisoning.

\section{EXPERIMENTAL SECTION}

Synthesis of Probe TP-SS. Compound 4 (0.0355 g, 0.1 $\mathrm{mmol})$, triphosgene $(0.090 \mathrm{~g}, 0.3 \mathrm{mmol})$ was dissolved in 50 $\mathrm{mL}$ anhydrous $\mathrm{CH}_{2} \mathrm{Cl}_{2}$ under $\mathrm{Ar}$ atmosphere. The mixture was suspended at $0{ }^{\circ} \mathrm{C}$, and then $1 \mathrm{~mL}$ DIPEA was added. The reaction lasted for $30 \mathrm{~min}$, and the color of the solution changed from purple to khaki. After the solvent was removed in vacuum, the obtained residue was dissolved in $50 \mathrm{~mL}$ of anhydrous $\mathrm{CH}_{2} \mathrm{Cl}_{2}$, and DIPEA (1 mL) and DMAP $(20 \mathrm{mg})$ were added. 2,2'-Dithiodiethanol $(0.030 \mathrm{~g}, 0.2 \mathrm{mmol})$ in anhydrous methylene chloride $(2 \mathrm{~mL})$ was added into above mixture. Then the reaction mixture was stirred at $25{ }^{\circ} \mathrm{C}$. TLC monitored the reaction until the starting material was completely consumed. The obtained solid residue was purified through silica gel chromatography (200-300 mesh) with gradient eluent of $\mathrm{CH}_{2} \mathrm{Cl}_{2}$ and $\mathrm{CH}_{3} \mathrm{OH}(100: 0$ to $85: 15 \mathrm{v} / \mathrm{v})$. The solvent was removed in vacuum to give a yellow solid (24.6 mg, yield 46\%). ${ }^{1} \mathrm{H}$ NMR (500 MHz, DMSO- $\left.d_{6}\right) \delta$ (ppm): 8.55 (s, 1H), $8.52(\mathrm{~s}, 2 \mathrm{H}), 8.19(\mathrm{~d}, J=1.5 \mathrm{~Hz}, 1 \mathrm{H})$, $8.18(\mathrm{~d}, J=1.5 \mathrm{~Hz}, 1 \mathrm{H}), 7.76(\mathrm{~s}, 1 \mathrm{H}), 7.74(\mathrm{~s}, 1 \mathrm{H}), 7.72(\mathrm{~s}$, $1 \mathrm{H}), 7.70(\mathrm{~s}, 1 \mathrm{H}), 7.56(\mathrm{~s}, 1 \mathrm{H}), 7.07$ (d, $J=2 \mathrm{~Hz}, 1 \mathrm{H}), 7.06$ (d, $J=2 \mathrm{~Hz}, 1 \mathrm{H}), 4.50(\mathrm{q}, J=7.5 \mathrm{~Hz}, 2 \mathrm{H}), 3.84(\mathrm{~s}, 1 \mathrm{H}), 3.62$ $(\mathrm{m}, 4 \mathrm{H}), 2.86(\mathrm{~s}, 3 \mathrm{H}), 2.79(\mathrm{~m}, 4 \mathrm{H}), 1.26(\mathrm{~s}, 6 \mathrm{H}), 1.06(\mathrm{t}, J=$ $7 \mathrm{~Hz}, 3 \mathrm{H})$. LC-MS (ESI $\left.{ }^{+}\right): m / z \mathrm{C}_{30} \mathrm{H}_{35} \mathrm{~N}_{2} \mathrm{O}_{3} \mathrm{~S}_{2}{ }^{+}$calcd. 535.74, found $\left[\mathrm{M}^{+}\right] 535.19$.

Transmission Electron Microscopy Observation. The cells were first fixed in fresh $2.5 \%$ glutaraldehyde for at least $4 \mathrm{~h}$ at 4 ${ }^{\circ} \mathrm{C}$ and then postfixed in $1 \%$ osmium tetroxide for $1.5 \mathrm{~h}$. Next, the samples were dehydrated in a gradient ethanol series and infiltrated with Epon812. The samples were then embedded and cured at $37^{\circ} \mathrm{C}$ for $12 \mathrm{~h}, 45^{\circ} \mathrm{C}$ for $12 \mathrm{~h}$, and $60^{\circ} \mathrm{C}$ for 24 h. Ultrathin sections were cut using an ultramicrotome (Lecia, Wetzlar, Germany) and stained with uranyl acetate and lead citrate before observation under the JEM-1400 transmission electron microscope (TEM; JEOL Ltd., Tokyo, Japan).

Western Blot Analysis. All of the cell samples were treated by different compounds and washed for three times before lysis. Protein extracts were prepared by suspending the cells in $200 \mu \mathrm{L}$ of RIPA lysis buffer containing 1\% PMSF (Solarbio, China). Protein concentrations were determined with a bicinchoninic acid (BCA) protein assay kit (Bioworld Technology, U.S.A.). Protein samples were extracted after centrifugation at $12500 \mathrm{~g}$ at $4{ }^{\circ} \mathrm{C}$ for $15 \mathrm{~min}$. Then, the protein was mixed with loading buffer $(4: 1)$ and heated at $95^{\circ} \mathrm{C}$ for 15 min. For Western blotting analysis, an equal amount of protein $(70 \mu \mathrm{g})$ was loaded in each well and subjected to sodium dodecyl sulfate-polyacrylamide gel electrophoresis (SDSPAGE). Separated proteins were then transferred from the gel to polyvinylidinene fluoride (Millipore, Bedford, MA, USA) membranes and blocked in $7 \%$ nonfat dry milk prepared in $1 \times$ TBST. The membranes were incubated with the primary antibodies overnight at $4{ }^{\circ} \mathrm{C}$. After washing primary antibodies with $1 \times \mathrm{TBST}$, the membranes were incubated with appropriate secondary antibodies for $1 \mathrm{~h}$ at room temperature. The membranes were developed using Super Enhancer ECL Kit (Shanghai Novland, China). The results were analyzed by Image $J$ to acquire the gray value of every bond.

Establishment of Mice Aging Models. BALB/c mice were obtained from Binzhou Medical University. Mice were selected according to the age and divided into three groups. Before treated with intracranial injection, the hair on the head was removed. Group a: the mice were 1 month old, which is equivalent to early childhood in humans. Group b: the mice were 12 months old, which is equivalent to middle-age in humans. Group c: the mice were 24 months old, which is equivalent to old-age in humans.

Establishing the Mice Model of Mercury Stress. BALB/c mice weighing about $20 \mathrm{~g}$ were provided by Binzhou Medical University. For the mice in group $\mathrm{b}, \mathrm{a} \mathrm{HgCl}_{2}$ solution $(18 \mathrm{mg} /$ $\mathrm{kg}, 0.2 \mathrm{~mL}$ ) was given orally by gavage for 3 days; for the mice in group c, a $\mathrm{HgCl}_{2}$ solution $(18 \mathrm{mg} / \mathrm{kg}, 0.2 \mathrm{~mL}$ ) and $\mathrm{Sec}$ $(0.485 \mathrm{mg} / \mathrm{kg})$ were given to the mice the same way. The control mice were given equivalent saline. Before being treated with intracranial injection, the hair on the head was removed.

\section{RESULTS AND DISCUSSION}

Design and Synthesis of Probe. Considering the advantages of two-photon functional fluorescent probes, we strived to conceive a novel two-photon ratiometric fluorescent probe for GPx detection. We adopted two-photon fluorophore TP-NH as chromophore due to its NIR fluorescence emission at $675 \mathrm{~nm} .{ }^{16}$ Sec that acted as the active site of GPx attracted our attention, and we selected $\mathrm{Sec}$ as a breakthrough to achieve GPx detection. Several one-photon fluorescent probes have 
been developed for Sec detection. ${ }^{17-22}$ However, they cannot achieve the detection of GPx, and they are limited to shallow depth of imaging. The cleavage of protein/organic disulfide is commonly occurred by selenium-sulfur exchange reactions to regulate redox states in vivo; thus, it is reasonable to study GPx based on selenium-sulfur exchange reaction. ${ }^{36,37}$ In our previous study, reactive disulfide (2,2'-dithiodiethanol) showed good selectivity for Sec without interferences from other biothiols. ${ }^{24}$ Therefore, we chose $2,2^{\prime}$-dithiodiethanol as the response unit for GPx detection, and the overall design strategy was shown in Scheme 1. We subtly integrated 2,2'-

Scheme 1. Reaction Mechanism of TP-SS for GPx Detection

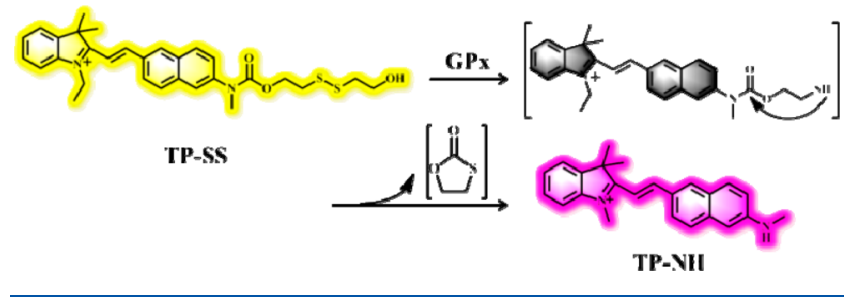

dithiodiethanol to two-photon fluorophore, TP-NH, to obtain a two-photon ratiometric fluorescent probe TP-SS for GPx detection. Once the disulfide bond was interrupted by GPx, TP-SS experienced a rapid intramolecular cyclization process to release fluorophore TP-NH, and TP-NH showed a large spectral red shift. Furthermore, ratiometric fluorescent probe TP-SS employed the ratio of the fluorescence spectra at two emission bands which can prevent the interference from various environmental conditions. ${ }^{11}$ The detailed synthesis routes of TP-SS are shown in the Supporting Information.

Spectroscopic Properties of TP-SS toward Gpx. Before GPx detection, we first investigated the spectral characteristics of TP-SS toward Sec under simulated physiological conditions. As shown in Figure S1, the spectral differences in different solvents were not obvious, thus we selected HEPES buffer for spectral studies. The probe TP-SS showed an absorption peak centered at $430 \mathrm{~nm}\left(\varepsilon=5.5 \times 10^{4} \mathrm{M}^{-1} \mathrm{~cm}^{-1}\right)$. After reaction with $\mathrm{Sec}$, the maximum absorption peak shifted to $545 \mathrm{~nm}(\varepsilon=$ $\left.1.7 \times 10^{5} \mathrm{M}^{-1} \mathrm{~cm}^{-1}\right)$. Then the fluorescence spectra of TP-SS toward Sec were detected (Figure 1a,b). TP-SS exhibited a maximum fluorescence emission peak at $570 \mathrm{~nm}$. After reaction with $\mathrm{Sec}$, the maximum fluorescence emission wavelength shifted from 570 to $675 \mathrm{~nm}$. We found that the ratio of fluorescence intensity $\left(F_{675 \mathrm{~nm}} / F_{570 \mathrm{~nm}}\right)$ increased with Sec increased (Figure S1c). There existed a linear relationship between logarithm of the ratio $\left(F_{675 \mathrm{~nm}} / F_{570 \mathrm{~nm}}\right)$ and $\mathrm{Sec}$ concentrations, and the regression equation was $\lg \left(F_{675 \mathrm{~nm}} /\right.$ $\left.F_{570 \mathrm{~nm}}\right)=0.08584[\mathrm{Sec}]-0.1461$, with $r=0.9828$ (Figure 1c). Then the time-dependent ratiometric fluorescence signal toward Sec was detected (Figure 1d). The results indicated that TP-SS can response to Sec rapidly based on sulfurselenium exchange reaction, and the reaction reached saturation within $140 \mathrm{~s}$. Thus, TP-SS was a promising tool for rapidly detection.

Encouraged by the results that TP-SS can be used for rapid detection of Sec, we attempted to detect GPx, a selenoprotein with Sec as the active site, using TP-SS. As shown in Figure 1e, TP-SS showed fluorescence emission centered at $570 \mathrm{~nm}$. After reaction with GPx, the maximum fluorescence emission peak shifted to $675 \mathrm{~nm}$ from $570 \mathrm{~nm}$ (Figure 1f). Then the reaction kinetics of TP-SS toward GPx was performed, and the results were shown in Figure 1g. The fluorescent signals displayed a manner of GPx concentration dependence. Ten U GPx (contain 2.5\% DTT, Sigma, G6137) converted almost all TP-SS to TP-NH within $5 \mathrm{~min}$. $\mathrm{Hg}^{2+}$ can strongly combine with selenium, and then we examined the effect of $\mathrm{Hg}^{2+}$ on GPx. As shown in Figure $1 \mathrm{~g}, 10 \mu \mathrm{M} \mathrm{Hg}^{2+}$ could greatly affected the reaction of GPx and TP-SS (green line). Thus, $\mathrm{Hg}^{2+}$ had a devastating effect on GPx.

High selectivity for GPx detection in the complex biological system was needed, and the interferences from reactive sulfur species (RSS) made the GPx detection more challenging because RSS exhibited nucleophilic characteristics like Sec, and they were at millimoles level such as GSH. Thus, we performed

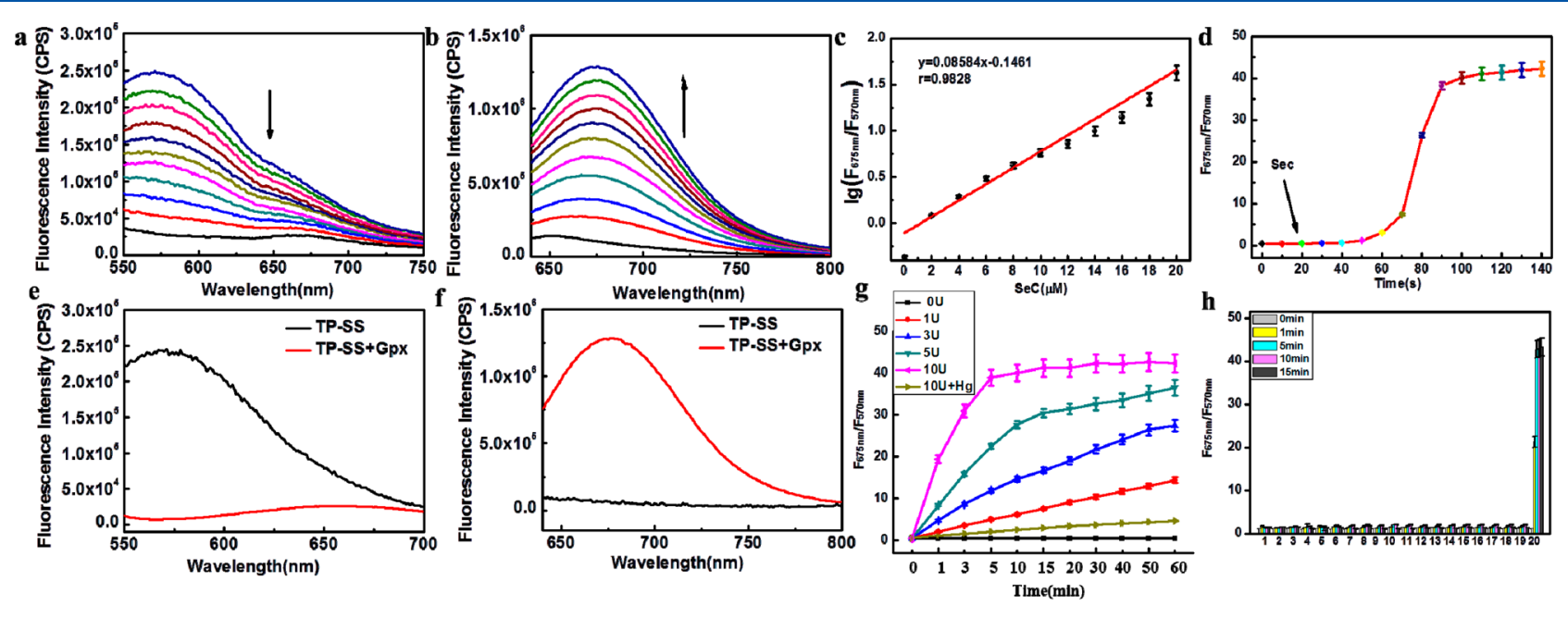

Figure 1. Fluorescence spectral of TP-SS $(10 \mu \mathrm{M})$ toward $\operatorname{Sec}(0-20 \mu \mathrm{M})$. (a) $\lambda_{\mathrm{ex}}=435 \mathrm{~nm}$. (b) $\lambda_{\mathrm{ex}}=550 \mathrm{~nm}$. (c) The linear relationship between $\lg \left(F_{675 \mathrm{~nm}} / F_{570 \mathrm{~nm}}\right)$ and Sec. (d) Reaction kinetic of probe toward Sec. The fluorescence spectra of TP-SS $(10 \mu \mathrm{M})$ toward GPx $(0-10 \mathrm{U})$. (e) $\lambda_{\mathrm{ex}}=435 \mathrm{~nm}$. (f) $\lambda_{\mathrm{ex}}=550 \mathrm{~nm}$. (g) Time-dependent ratio fluorescence signal of TP-SS $(10 \mu \mathrm{M})$ toward GPx. (h) Selectivity of TP-SS toward various interfering species. 1, blank; 2, $20 \mu \mathrm{M}$ ascorbic acid; 3, $20 \mu \mathrm{M} \mathrm{N}$-acetyl-L-cysteine; 4, $20 \mu \mathrm{M} \mathrm{NaHS;} \mathrm{5,} 200 \mu \mathrm{M}$ Cys; 6, $20 \mu \mathrm{M}$ GSSH; 7, 20 $\mu \mathrm{M}$ Hcy; 8, $20 \mu \mathrm{M}$ Cys-SSH; 9, $200 \mu \mathrm{M} \mathrm{NO}^{2-} ; 10,1 \mathrm{mM} \mathrm{H}_{2} \mathrm{O}_{2} ; 11,200 \mu \mathrm{M} \mathrm{ClO}^{-} ; 12,200 \mu \mathrm{M} \mathrm{O}_{2}^{--} ; 13,1 \mathrm{mM} \mathrm{K}^{+} ; 14,1 \mathrm{mM} \mathrm{Ca}^{2+} ; 15,1 \mathrm{mM}^{-}$ $\mathrm{Zn}^{2+}$; $16,1 \mathrm{mM} \mathrm{Cu}^{2+}$; $17,1 \mathrm{mM} \mathrm{H}_{2} \mathrm{PO}_{4}^{-} ; 18,1 \mathrm{mM} \mathrm{Br}^{-}$; $19,1 \mathrm{mM} \mathrm{CO}_{3}^{2-}$; 20, $10 \mathrm{U} \mathrm{GPx}$. 
selectivity tests for GPx detection carefully among various interfering species. As shown in Figure 1h, biothiols and other active species can not interfere with GPx detection. In addition, we carefully performed interference test with GSH. As shown in Figure S2, we found that GSH and DTT could not interfere with GPx detection within $10 \mathrm{~min}$. And the reasons can be attributed to the following aspects. The $\mathrm{p} K_{\mathrm{a}}$ of Sec was about 5.2, which was much lower than that of biothiol $\left(\mathrm{GSH}, \mathrm{p} K_{\mathrm{a}}=8.75\right.$ and Cys, $\left.\mathrm{p} K_{\mathrm{a}}=8.29\right) .{ }^{23}$ Under physiological $\mathrm{pH}$ (7.40), Sec almost existed as the fully deprotonated anion form $\left(\mathrm{RSe}^{-}\right)$, while GSH and cysteine (Cys) existed as the protonated anion form (RSH), indicating that Sec had higher nucleophilic properties and reactive activity than $\mathrm{GSH}$ and Cys. Thus, we can avoid the interference from GSH and other interfering species by rapid detection within $10 \mathrm{~min}$, which was also in line with the fast detection requirements of GPx. Furthermore, the stability of TP-SS was detected, and the results indicated that TP-SS was a stable tool for GPx detection (Figure S4).

Exploring Catalysis Cycle of GPx/GSH Redox Pool in Vitro. Encouraged by the GPx detection, we attempted to explore the catalytic cycle of the $\mathrm{GPx} / \mathrm{GSH}$ redox pool in vitro using TP-SS. Under physiological conditions, Sec, the active

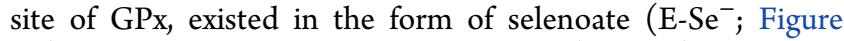
$2 \mathrm{~A})$. After reaction with organic peroxide $(\mathrm{ROOH}), \mathrm{E}^{-} \mathrm{Se}^{-}$can

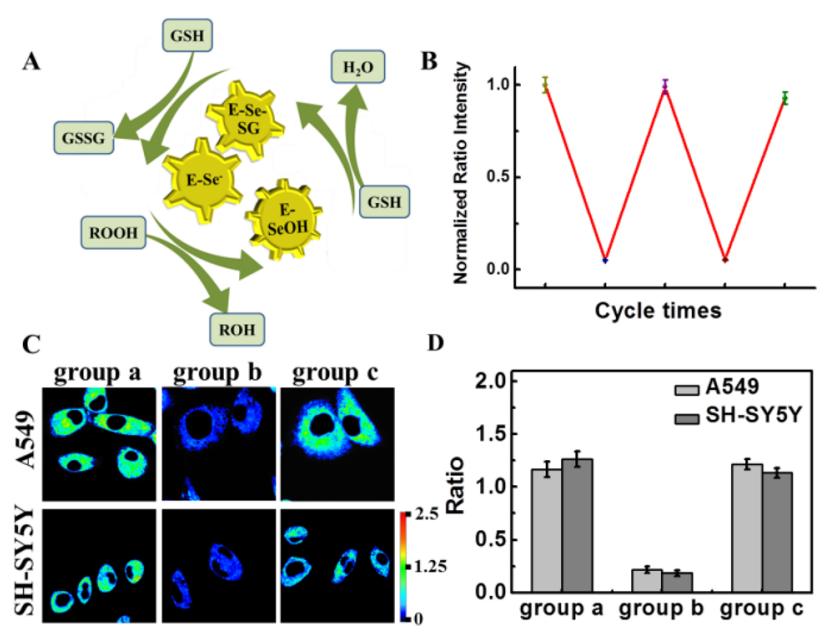

Figure 2. Exploring the catalysis cycle of GPx/GSH redox pool with TP-SS. (A) Scheme of catalytic cycle of GPx/GSH redox pool. (B) Normalized fluorescence responses of TP-SS during the redox cycles. (C) GPx detection in living cells: group a, $10 \mu \mathrm{M}$ TP-SS (for $10 \mathrm{~min}$ ); group b, $10 \mu \mathrm{M} \mathrm{Hg}^{2+}$ and $10 \mu \mathrm{M}$ TP-SS; group c, $10 \mu \mathrm{M} \mathrm{Hg}^{2+}, 10 \mu \mathrm{M}$ Sec and $10 \mu \mathrm{M}$ TP-SS. (D) Relative ratio fluorescence intensities of TP-SS in (C).

be oxidized to the selenic acid form (E-SeOH), and this redox cycle can be maintained and cycled by GSH reduced $\mathrm{E}-\mathrm{SeOH}$ to $\mathrm{E}-\mathrm{Se}^{-25}$. We then simulated the redox cycle of GPx in the simulated physiological conditions (10 mM HEPES, $\mathrm{pH} 7.4$ ), and all of the signals were recorded within $5 \mathrm{~min}$. We used $\mathrm{H}_{2} \mathrm{O}_{2}$ to simulate oxidant $\mathrm{ROOH}$, and $\mathrm{GSH}$ was used as the reduction reagent. As shown in Figure 2B, TP-SS $(10 \mu \mathrm{M})$ first reacted with GPx $(10 \mathrm{U})$, and highly ratio fluorescence signals were captured. All tests in the other groups were treated as the first group. Then, $200 \mu \mathrm{M} \mathrm{H}_{2} \mathrm{O}_{2}$ was added, and the ratio fluorescence signals were decreased, which indicated that GPx was oxidized by $\mathrm{H}_{2} \mathrm{O}_{2}$. Next, we used $500 \mu \mathrm{M}$ GSH to reduce $\mathrm{GPx}$, and the ratio fluorescence signal was restored. The results demonstrated that TP-SS can be used for tracking the GPx/ GSH redox pool in vitro. The experimental results from Figure $2 \mathrm{~B}$ indicated that the redox cycle could be repeated at least two times.

Then we detected GPx in living cells. Before cell tests, we performed MTT assays to evaluate the cytotoxicity of TP-SS. The high cell viability in Figure S5 demonstrated that TP-SS had low cytotoxicity. As shown in Figure 2C, the cells in group a were incubated with $10 \mu \mathrm{M}$ TP-SS, and bright fluorescence signals were captured. The results confirmed that TP-SS can respond to GPx in living cells. All experiments in the other groups were treated as group a. Cells in group b were incubated with $10 \mu \mathrm{M} \mathrm{Hg}^{2+}$ for $2 \mathrm{~h}$, and we can observe a weak fluorescence ratio. We supposed that $\mathrm{Hg}^{2+}$ decreased the level of GPx from two path ways. On the one hand, $\mathrm{Hg}^{2+}$ can combine with GPx and decrease the level of GPx directly. On the other hand, $\mathrm{Hg}^{2+}$ caused oxidative stress, which further decreased GPx. Then the cells in group $\mathrm{c}$ were pretreated with $10 \mu \mathrm{M} \mathrm{Hg}^{2+}$ for $2 \mathrm{~h}$, and $10 \mu \mathrm{M}$ Sec was added. Increased fluorescence signals were observed. The relative ratio fluorescence intensities in Figure 2C were shown in Figure 2D. These results demonstrated that TP-SS can be applied for GPx detection in living cells.

Detecting the Catalytic Cycle of GPx/GSH Redox Pool and the Concentration Fluctuation of GPx in the Aging Model. Inspired by the results from the in vitro catalytic cycle, we further simulated the catalytic cycle of the GPx/GSH redox pool in SH-SY5Y cells. During aging, oxidative stress was abnormally increased and associated with many diseases such as Parkinson's disease. Then we established the aging cell model with $\mathrm{H}_{2} \mathrm{O}_{2}{ }^{26}$ As shown in Figure 3A, the cells in group a were treated with $10 \mu \mathrm{M}$ TP-SS for $10 \mathrm{~min}$, and GPx triggered bright ratio fluorescence signals. All cells in the other groups were treated as group a. Then we used $\mathrm{H}_{2} \mathrm{O}_{2}$ to simulate oxidative stress during aging in the following groups. The cells in group $\mathrm{b}$ were treated with $\mathrm{H}_{2} \mathrm{O}_{2}(200 \mu \mathrm{M})$ for $1 \mathrm{~h}$, and the ratio fluorescence signals were decreased greatly. The results indicated that GPx was oxidized by $\mathrm{H}_{2} \mathrm{O}_{2}$, which led to the ratio fluorescence signals decreasing. The cells in group $\mathrm{c}$ were pretreated as described in group b, and then $500 \mu \mathrm{M}$ GSH was added. A bright ratio fluorescence signal was observed. The fluorescence recovery in group c represented that GSH reduced GPx to its selenoate form $\left(\mathrm{E}_{-} \mathrm{Se}^{-}\right)$, and GPx triggered the ratio fluorescence signals to increase. The cells in group $\mathrm{d}$ were treated as described in group c, and then $200 \mu \mathrm{M}$ $\mathrm{H}_{2} \mathrm{O}_{2}$ was added. As expected, we observed a decrease in the ratio signal. After treated as described in group $\mathrm{d}$, the cells in group e were incubated with $500 \mu \mathrm{M} \mathrm{GSH}$, and the ratio signal had risen. Repeated experiments also showed similar results. Flow cytometry analysis has been recognized as a highthroughput analytical technique and has been widely used to analyze large numbers of samples. These results were further confirmed by flow cytometry analysis (Figure 3B), and the results from confocal microscopy were consistent with that from flow cytometry. The quantitative analysis of ratio fluorescence intensity for Figure 3A,B are shown in Figure 3F,G. The results demonstrated that TP-SS could track the catalytic cycle of GPx/GSH redox pool in living cells.

Oxidative stress can induce cell damage and further lead to $\mathrm{Ca}^{2+}$, which is stored in mitochondria and flows to the cytoplasm. ${ }^{27-29}$ Then the $\mathrm{Ca}^{2+}$ concentrations were detected using Fluo 4-AM, and the results are shown in Figure 3C. The $\mathrm{Ca}^{2+}$ concentrations can be sorted as groups $\mathrm{d}>\mathrm{e}>\mathrm{b}>\mathrm{c}>\mathrm{a}$, 


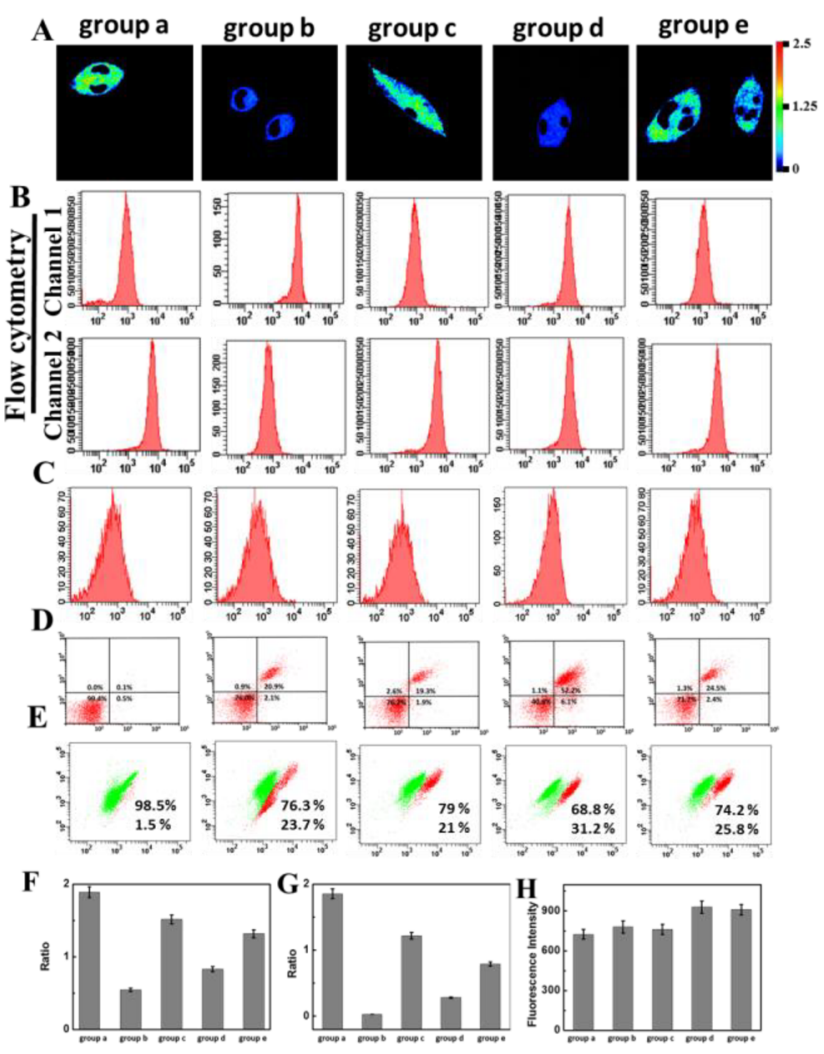

Figure 3. (A) Simulating catalytic cycle of GPx/GSH redox pool in SH-SY5Y cells. (B) Flow cytometry analysis. (C) $\mathrm{Ca}^{2+}$ concentration analysis. (D) Apoptosis analysis: viable cells (AnnexinV-/7-AAD-), early apoptosis (AnnexinV+/7-AAD-), late apoptosis (AnnexinV+/7$\mathrm{AAD}+$ ), and necrosis (AnnexinV-/7-AAD+). (E) $\Delta \Psi \mathrm{m}$ analysis by JC-1. (F) Quantitative analysis of ratio fluorescence intensity in panel A. (G) Quantitative analysis of ratio fluorescence intensity in panel B. $(\mathrm{H})$ Quantitative analysis of $\mathrm{Ca}^{2+}$ concentration in Figure 3C. The data were shown as mean ( \pm s.d.; $n=5)$. which indicated that the cell damages can be sorted as groups $\mathrm{d}$ $>\mathrm{e}>\mathrm{b}>\mathrm{c}>\mathrm{a}$. The quantitative analysis of $\mathrm{Ca}^{2+}$ concentrations was shown in Figure $3 \mathrm{H}$. Then we used an Annexin V/7-AAD Apoptosis Detection Kit to confirm the cell damage, and the results were consistent with the results from $\mathrm{Ca}^{2+}$ detection (Figure 3D). Mitochondrial membrane potential $\left(\Delta \Psi_{\mathrm{m}}\right)$ was another indicator for evaluating the cell damage, and the outflow of $\mathrm{Ca}^{2+}$ would cause the $\Delta \Psi_{\mathrm{m}}$ to collapse. $\Delta \Psi_{\mathrm{m}}$ can be detected using JC-1, and the decreasing green/red fluorescence intensity ratio revealed the decrease of $\Delta \Psi_{\mathrm{m}}$, which represented the increasing cell damage. As shown in Figure 3E, the cell damage could be sorted as groups $\mathrm{d}>\mathrm{e}>$ $\mathrm{b}>\mathrm{c}>\mathrm{a}$. These results from cell damage detection were consistent, and the experimental results illustrated that we can evaluate oxidative stress through GPx detection using TP-SS.

Encouraged by GPx detection in living cells, we further performed GPx detection in vivo. Studies showed that the activity of GPx was decreased during aging, especially in patients with Parkinson's disease. ${ }^{30}$ We then performed GPx detection in aging mice models. As shown in Figure 4, the mice were divided into three groups according to age: 1,12 , and 24 months old. The hair on the mice heads was removed, and mice were treated with intracranial injection of TP-SS, and then the fluorescence signals were collected (Figure 4a). The ratio fluorescence signals decreased with age, which indicated that the activity of GPx decreased with age. Then the isolated organs including heart, liver, spleen, lungs, kidneys, and brain were obtained for imaging (Figure $4 \mathrm{~b}$ ). We can capture the fluorescence signals only in brains. The fluorescence quantitative analysis of Figure 4a,b are shown in Figure 4d,e. Furthermore, the fresh brain tissue slices were obtained as quickly as possible for fluorescence imaging using $10 \mu \mathrm{M}$ TPSS (Figure 4c). The ratio fluorescence intensities could be sorted as $1>12>24$ months and the results demonstrated that the activity of GPx was decreased during aging. The results from Figure 4 were consistent, indicating TP-SS was a promising chemical tool for GPx detection in vivo. ${ }^{35}$

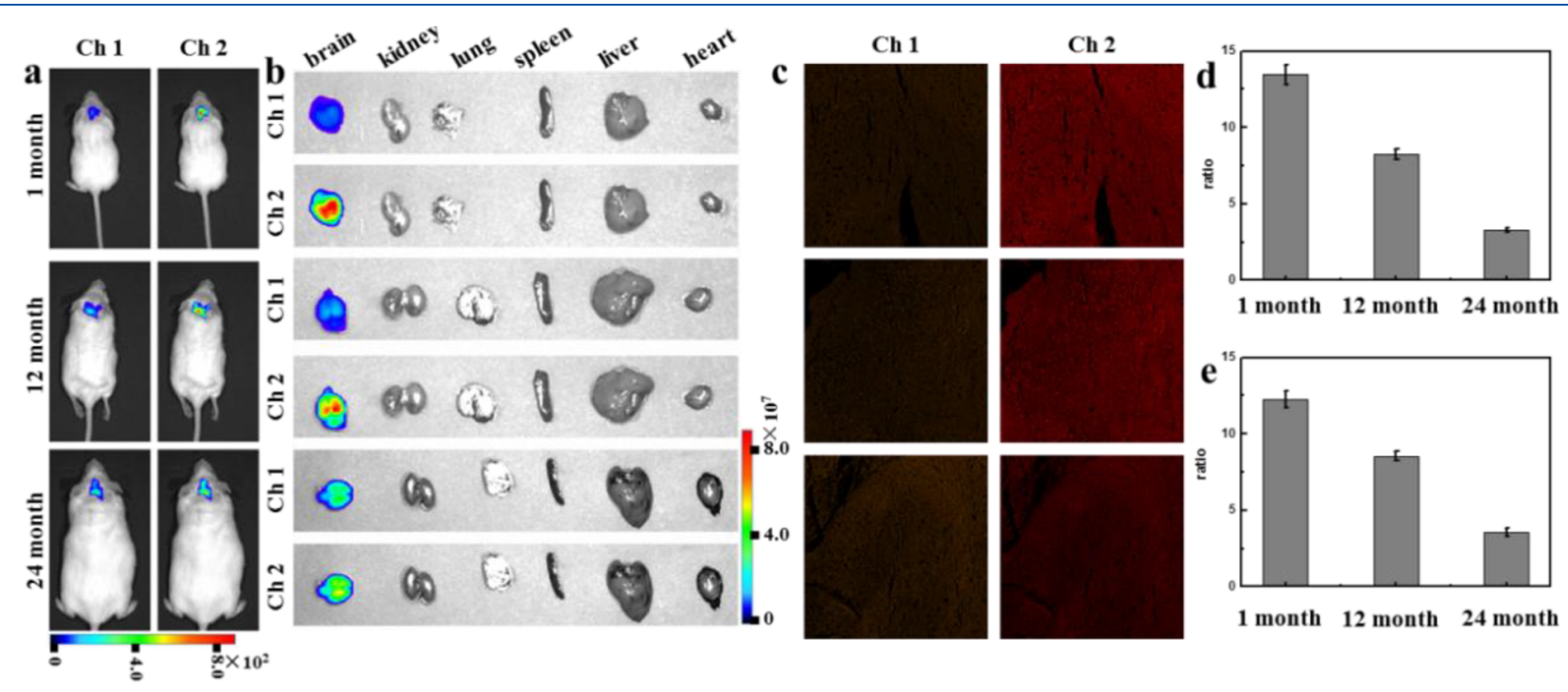

Figure 4. GPx detection in aging mice model. (a) In vivo fluorescence imaging of GPx. (b) In vitro imaging of GPx in separated organs. (c) GPx detection in fresh brain slices from panel a. (d) The relative fluorescence ratio for panel a. (e) The fluorescence ratios for panel b. The mice were intracranial injected with TP-SS $\left(100 \mu \mathrm{M}, 50 \mu \mathrm{L}\right.$ in 1:99 DMSO/saline, v/v) for $30 \mathrm{~min}$. Fluorescence signals were collected in channel 1 (Ch1, $\lambda_{\text {ex }}$ : $\left.465 \mathrm{~nm} ; \lambda_{\mathrm{em}}: 600 \mathrm{~nm}\right)$ and channel $2\left(\mathrm{Ch} 2, \lambda_{\mathrm{ex}}: 535 \mathrm{~nm} ; \lambda_{\mathrm{em}}: 680 \mathrm{~nm}\right)$. The hair on the mice heads has been removed. 

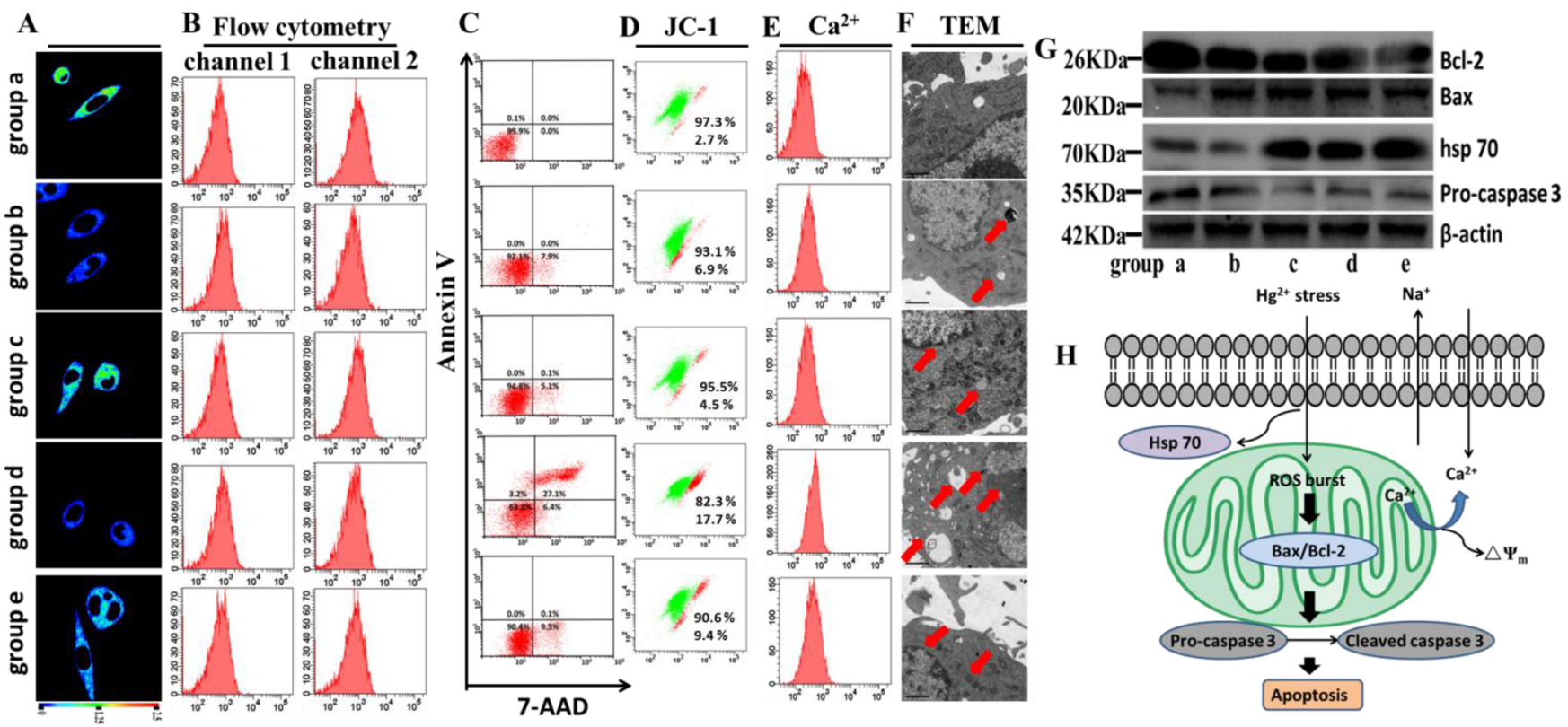

Figure 5. (A) Tracking the catalytic cycle of GPx/GSH redox pool using confocal microscopy. (B) Flow cytometry analysis. (C) Apoptosis analysis. (D) $\Delta \Psi_{\mathrm{m}}$ analysis by JC-1. (E) $\mathrm{Ca}^{2+}$ concentration analysis using Fluo 4-AM. (F) TEM observation (scale bars: $1 \mu \mathrm{m}$ ). (G) Western blotting analysis of Bcl-2, Bax, hsp70 and Pro-caspase 3, $\beta$-actin was used as a loading control. (H) Scheme of signal transduction pathways of $\mathrm{Hg}^{2+}$ induced oxidative stress.

Tracking the GPx/GSH Redox Pool in the $\mathrm{Hg}^{2+}$ Induced Mercury Poisoning Model. $\mathrm{Hg}^{2+}$ as toxic heavy metal ion has severe bioaccumulation and protein targeting. Studies have shown that the antagonism of $\mathrm{Hg}^{2+}$ and selenoprotein such as $\mathrm{Sec}$ is also a detoxification mechanism for mercury poisoning. ${ }^{31,32}$ Therefore, we guess that $\mathrm{Hg}^{2+}$ can also affect the enzyme activity of GPx. Exploring the effect of $\mathrm{Hg}^{2+}$ on GPx/GSH redox pool is much more valuable for further elucidating the physiological activities related to mercury poisoning and GPx/GSH redox pool. As shown in Figure 5A, the cells in group a were treated with $10 \mu \mathrm{M}$ TP-SS for $10 \mathrm{~min}$ and bright ratio fluorescence signals can be observed. All cells in the other groups were treated as group a. The cells in group b were treated with $10 \mu \mathrm{M} \mathrm{Hg}^{2+}$ for $2 \mathrm{~h}$, and decreased ratio fluorescence signals can be observed. Then GSH $(500 \mu \mathrm{M}, 1 \mathrm{~h})$ was used as a reduction reagent in group c to promote the redox cycle of GPx/GSH redox pool, and increased ratio fluorescence signals were observed. However, the fluorescence signals in group $\mathrm{c}$ were weaker than that from group a. We speculated that some $\mathrm{Hg}^{2+}$ reacted with the active site of GPx which caused irreversible enzyme inactivation. Next cells in groups $\mathrm{d}$ and e were processed with the same operation to repeat the catalytic cycle of GPx/GSH redox pool. The results indicated that the catalytic cycle of GPx/GSH redox pool can be repeated at least two times in cells. The ratio fluorescence signals in Figure 5A were confirmed by flow cytometry, and the results were consistent (Figure 5B). The quantitative analysis of the fluorescence intensity ratio of Figure 5A,B is shown in Figure S9a,b.

The cell damages caused by $\mathrm{Hg}^{2+}$ were evaluated by an Annexin V/7-AAD Apoptosis Detection Kit. As shown in Figure 5C, cell damage became much more sever with the number of cycles increased, and it can be sorted as groups $d>$ $\mathrm{e}>\mathrm{b}>\mathrm{c}>\mathrm{a}$. Increased cell damage would lead to a decrease in $\Delta \Psi_{\mathrm{m}}$, and we detected $\Delta \Psi_{\mathrm{m}}$ using a commercial probe JC-1. As shown in Figure 5D, the $\Delta \Psi_{\mathrm{m}}$ decreased with increased cell damage (the ratio of green/red decreased). Then we detected the concentration of $\mathrm{Ca}^{2+}$ with Fluo 4-AM (Figure 5E). The quantitative analysis of $\mathrm{Ca}^{2+}$ concentrations was shown in Figure S9c. We found that the concentration of $\mathrm{Ca}^{2+}$ increased with increased cell damage. TEM was further used to observe the ultrastructure of cells (Figure 5F). We observed intact membrane structures and regular organelles in group a. However, the membrane structure became blurred and the organelle structure was also deformed to varying degrees in the experimental groups (group $\mathrm{b}-\mathrm{e}$ ). These results demonstrated that $\mathrm{Hg}^{2+}$ destroyed the active site of GPx and further caused cell damages. We speculated that the selenium-containing antidote might be more effective than the sulfur-containing antidote.

$\mathrm{Hg}^{2+}$ destroyed the GPx/GSH redox pool and further caused ROS to burst as well as affected the signaling pathways associated with oxidative stress. We next detected the protein signaling pathways associated with oxidative stress using Western blot. Severe cell damage led to apoptosis, while protein Bcl-2 can inhibit apoptosis factor and prevent programmed cell death. ${ }^{33}$ However, overexpressed Bax can inhibit the activity of $\mathrm{Bcl}-2$, and the ratio of $\mathrm{Bcl}-2$ to $\mathrm{Bax}$ determined survival or death following apoptosis stimulus. ${ }^{33}$ Then the two proteins were detected by Western blot (Figure $5 G)$. We found that Bax was up-regulated and Bcl-2 was downregulated with cell damage worsen. Hsp 70 was a protective protein that responds to environmental stress in vivo. ${ }^{34} \mathrm{We}$ then detected the expression of hsp 70, and the results showed that hsp 70 was upregulated to protect cells from mercury poisoning. A series of unfavorable stimuli would eventually lead to caspase 3 activation and cell death. We evaluated caspase 3 by detecting pro-caspase 3 , and we found that mercury poisoning can down-regulate pro-caspase 3 . These results further confirmed that $\mathrm{Hg}^{2+}$ can destroy GPx which is involved in the GPx/GSH redox pool and further lead to oxidative stress damage. Thus, mercury poisoning can activate 

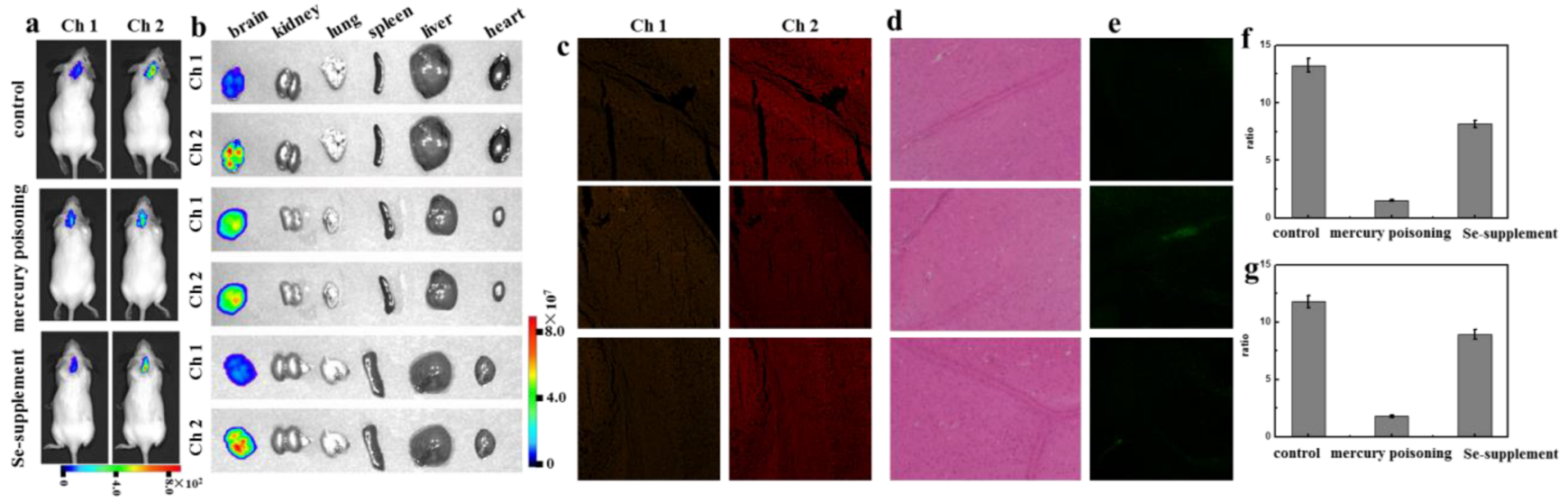

Figure 6. GPx detection in mercury poisoning mice model. (a) In vivo fluorescence imaging of GPx. (b) In vitro fluorescence imaging of GPx in separated organs (brain, kidney, lung, spleen, liver and heart). (c) GPx detection in fresh brain slices from panel a. (d) H\&E staining. (e) TUNEL staining. (f) The relative fluorescence ratios for panel a. (g) The relative fluorescence ratios for panel $b$. The hair on the mice heads has been removed.

the caspase-dependent apoptotic pathway, and organisms can initiate a protection mechanism, upregulating hsp 70 to protect themselves. The signal path mechanism in mercury poisoning was shown in Figure $5 \mathrm{H}$.

Then we performed GPx detection and evaluated the protective role of GPx in mercury poisoning mice models. The mice in the first group were given $0.2 \mathrm{~mL}$ of saline orally by gavage as a control. Then mice in the second group were given $0.2 \mathrm{~mL}$ of $\mathrm{HgCl}_{2}$ solution $(18 \mathrm{mg} / \mathrm{kg}$ ) for 3 days as the model group of mercury poisoning. The mice in the third group were given $0.2 \mathrm{~mL}$ of $\mathrm{HgCl}_{2}$ solution $(18 \mathrm{mg} / \mathrm{kg})$ and $\mathrm{Sec}(0.485$ $\mathrm{mg} / \mathrm{kg}$ ) for 3 days as the treatment group of mercury poisoning (Se-supplement). Then the fluorescence imaging of GPx in the brain was performed through intracranial injection. As shown in Figure 6a, the mice in the control group showed strong ratio fluorescence signals, which indicated that GPx was maintained at a normal level. However, the ratio fluorescence signals in the mercury poisoning mice were decreased dramatically. We supposed that $\mathrm{Hg}^{2+}$ with strong electrophilicity and cytotoxicity can destroy the structure of GPx and reduce the enzyme activity of GPx. After treatment with Sec, the fluorescence signals increased. Then fluorescence detection was performed on the isolated organs and only brains showed bright fluorescence signals (Figure 6b). The relative fluorescence quantitative analysis of Figure $6 a, b$ is shown in Figure 6f,g. The results were consistent. Furthermore, fresh brain tissue slices were obtained for fluorescence imaging (Figure 6c). Then we examined the histopathological morphology of the brain tissues by H\&E staining. As shown in Figure $6 \mathrm{~d}$, the brain tissue in the control group shaped rule and closely arranged. However, the morphology of the tissue in the second group became irregular and the arrangement became loose. After Sec treatment, we found that the damage of the brain tissue was relieved. When the cells were apoptotic, cells would activate some endonuclease enzymes to cut off the genomic DNA. Next TUNEL staining was performed for assessment of cell apoptosis by staining the disrupted DNA (Figure 6e). We found that the fluorescence signals were most obvious in the mercury poisoning group, and the fluorescence signals in the Se-supplement group were decreased. The results indicated that $\mathrm{Hg}^{2+}$ caused serious tissue damage in organisms, and the tissue damage in the mercury poisoning group was most sever. Sec can effectively alleviate tissue damages caused by mercury poisoning through selenium-mercury antagonism. These results revealed that we can assess tissue damage by TPSS to observe changes in GPx concentration during mercury poisoning. The research provides us a novel perspective for the study of the mercury poisoning mechanism, and TP-SS can help to assess mercury poisoning.

Encouraged by GPx detection using TP-SS in vivo, we then performed GPx detection in deep brain tissue. We first performed GPx detection in living cells using a two-photon laser confocal microscope. As shown in Figure S12, we can capture complete fluorescence signals about cell activities at each layer of the whole cell. Then the brain tissue samples from Figure 6a were manipulated as quickly as possible for quick detection using two-photon microscopy (Figure S14). The brain tissue samples were incubated with TP-SS $(10 \mu \mathrm{M})$ for $10 \mathrm{~min}$, and the fluorescence signals were captured by twophoton microscopy. We can observe vivid fluorescence signals at different depths with TP-SS in the control group, which represented the normal concentration of GPx in the brain. The fluorescence signals in the mercury poisoning group were much weaker than that from the control group. We considered that $\mathrm{Hg}^{2+}$ could pass through the blood-brain barrier and destroy GPx in the brain. We can observe increased fluorescence signals in the Se-supplement group. These results indicated that TP-SS can enable in situ, real-time, noninvasive fluorescence imaging of GPx at different tissue depths including the imaging depth up to $100 \mu \mathrm{m}$.

\section{CONCLUSION}

In summary, we have developed the first two-photon ratiometric fluorescent probe (TP-SS) for GPx detection. With the help of TP-SS, we explored the reversible catalysis cycle of the GPx/GSH redox pool in living cells and in vivo. We first investigated the concentration fluctuation of GPx in aging cell and mice models, and the results demonstrated that the activity of GPx was decreased during aging. The antioxidant mechanism of the GPx/GSH redox pool in the mercury poisoning cell and mice models was also studied. The results illustrated that $\mathrm{Hg}^{2+}$ might destroy the active site of GPx and further resulted in oxidative stress as well as an activated apoptotic pathway through inducing ROS burst, thereby aggravating the organism damage. GPx/GSH redox pool can effectively relief oxidative stress using GSH as the 
obligate cosubstrate to reduce ROS and the protein pathway hsp70 was upregulated to protect organisms. We further studied the concentration fluctuation of GPx in deep brain tissues, with an imaging depth up to $100 \mu \mathrm{m}$. We believe that the novel probe is a promising chemical tool for exploring the physiological functions of GPx and the cellular redox homeostasis events, which will be beneficial for clarifying the physiological and pathological significance of the GPx/GSH redox pool. Exploring the cyclic mechanism of the GPx/GSH redox pool can provide important insights into the biological basis of increased oxidative stress in the elderly, mercury poisoning in humans, as well as potential targets for clinical intervention. These studies provide us a novel perspective for the study of the mercury poisoning mechanism. We anticipate that TP-SS can facilitate the development of GPx-targeting tools.

\section{ASSOCIATED CONTENT}

\section{S Supporting Information}

The Supporting Information is available free of charge at https://pubs.acs.org/doi/10.1021/acs.analchem.9b04381.

Experimental supplementary methods for chemical synthesis and characteristic of compounds, supplementary experiments, and data (PDF)

\section{AUTHOR INFORMATION}

\section{Corresponding Author}

*E-mail: 1xchen@yic.ac.cn.

\section{ORCID}

Liangwei Zhang: 0000-0001-5317-0087

Lingxin Chen: 0000-0002-3764-3515

\section{Notes}

The authors declare no competing financial interest.

\section{ACKNOWLEDGMENTS}

We thank the National Nature Science Foundation of China (Nos. 21976209, 21778026, and 21575159), the program of Youth Innovation Promotion Association, CAS (Grant 2019217), and Taishan Scholar Project Special Funding (No. ts20190962).

\section{REFERENCES}

(1) Arthur, J. R. Cell. Mol. Life Sci. 2001, 57, 1825.

(2) Patching, S. G.; Gardiner, R. H. E. J. Trace Elem. Med. Biol. 1999, 13, 193.

(3) Penglase, S.; Hamre, K.; Ellingsen, S. Free Radical Biol. Med. 2014, 75, 95-104.

(4) Hazelton, G. A.; Lang, C. A. Mech. Ageing Dev. 1985, 29, 71.

(5) Huang, Y.; Ge, D. Y.; Zong, H. Catalysts 2017, 7, 282.

(6) Rahmanto, A. S.; Davies, M. J. IUBMB Life 2012, 64, 863-871.

(7) Weekley, C. M.; Harris, H. H. Chem. Soc. Rev. 2013, 42, 88708894

(8) Kadota, Y.; Suzuki, S.; Ideta, S. Eur. J. Pharmacol. 2010, 626, $166-170$.

(9) Mauriz, J. L.; Molpeceres, V.; García-Mediavilla, M. V. J. Pineal Res. 2007, 42, 222-230.

(10) Espinoza, S. E.; Guo, H.; Fedarko, N. J. Gerontol., Ser. A 2008, 63, 505-509.

(11) Wang, Y.; Gao, M.; Chen, Q. Anal. Chem. 2018, 90, 97699778.

(12) Branco, V.; Canário, J.; Lu, J. Free Radical Biol. Med. 2012, 52, 781-793.
(13) Molina-Jiménez, M. F.; Sánchez-Reus, M. I.; Cascales, M. Toxicol. Appl. Pharmacol. 2005, 209, 214-225.

(14) Ballihaut, G.; Mounicou, S.; Lobinski, R. Anal. Bioanal. Chem. 2007, 388, 585-591.

(15) Encinar, J. R.; Schaumlöffel, D.; Ogra, Y. Anal. Chem. 2004, 76, 6635-6642.

(16) Wang, Y.; Zhang, L.; Zhang, S. Anal. Chem. 2019, 91, 78127818.

(17) Maeda, H.; Katayama, K.; Matsuno, H. Angew. Chem., Int. Ed. 2006, 45, 1810-1813.

(18) Sun, Q.; Yang, S. H.; Wu, L. Anal. Chem. 2016, 88, 6084-6091.

(19) Feng, W.; Li, M.; Sun, Y. Anal. Chem. 2017, 89, 6106-6112.

(20) Chen, H.; Dong, B.; Tang, Y. Chem. - Eur. J. 2015, 21, 1169611700 .

(21) Zhang, B.; Ge, C.; Yao, J. J. Am. Chem. Soc. 2015, 137, 757769.

(22) Kong, F.; Hu, B.; Gao, Y. Chem. Commun. 2015, 51, 31023105.

(23) Huber, R. E.; Criddle, R. S. Arch. Biochem. Biophys. 1967, 122, 164.

(24) Han, X.; Song, X.; Yu, F. Adv. Funct. Mater. 2017, 27, 1700769.

(25) Patching, S. G.; Gardiner, R. H. E. J. Trace Elem. Med. Biol. 1999, 13, 193-214.

(26) Guo, G.; Ma, L.; Jiang, B.; Yi, J.; Tong, T.; Wang, W. J. J. Cell. Biochem. 2010, 109, 1000-1005.

(27) Nicotera, P.; Bellomo, G.; Orrenius, S. Annu. Rev. Pharmacol. Toxicol. 1992, 32, 449-470.

(28) Orrenius, S.; Burkitt, M. J.; Kass, G. E. N. Ann. Neurol. 1992, 32, S33-S42.

(29) Kruidering, M.; Van de Water, B.; de Heer, E.; Mulder, G. J.; Nagelkerke, J. F. J. Pharmacol. Exp. Ther. 1997, 280, 638-649.

(30) Kish, S. J.; Morito, C.; Hornykiewicz, O. Neurosci. Lett. 1985, $58,343-346$.

(31) Aschner, M.; Aschner, J. L. Neurosci. Biobehav. Rev. 1990, 14, 169-176.

(32) Raymond, L. J.; Ralston, N. V. C. Seychelles Medical and Dental Journal 2004, 7, 72-77.

(33) Oltval, Z. N.; Milliman, C. L.; Korsmeyer, S. J. Cell 1993, 74, 609-619.

(34) Mićović, V.; Bulog, A.; Kučić, N. Environ. Toxicol. Pharmacol. 2009, 28, 439-447.

(35) Cand, F.; Verdetti, J. Free Radical Biol. Med. 1989, 7, 59-63.

(36) Kharma, A.; Grman, M.; Misak, A. Molecules 2019, 24, 1359.

(37) Wang, Y.; Gao, M.; Liao, C.; Yu, F.; Chen, L. Sens. Actuators, B 2019, 301, 127038. 